\title{
Enhanced thermoelectric performance of chalcopyrite nanocomposite via co-milling of synthetic and natural minerals
}

Peter Baláža , Erika Dutkováa*, Petr Levinskýb, Nina Daneuc, Lenka Kubíčkováb,d, Karel Knížek ${ }^{\mathrm{b}}$, Matej Baláža , Jiří Navrátil ${ }^{b, e}$, Jana Kašparováe, Vadim Ksenofontov ${ }^{d}$, Angela Möller ${ }^{d}$, Jiří Hejtmánek ${ }^{b}$

aInstitute of Geotechnics, Slovak Academy of Sciences, Watsonova 45, 04001 Košice, Slovakia

bInstitute of Physics of the Czech Academy of Sciences, Cukrovarnická 10/112, 16200 Prague, Czech

Republic

'Jozef Stefan Institute, Jamova cesta 3, S1-1000 Ljubljana, Slovenia

${ }^{d}$ Department of Chemistry, Johannes Gutenberg-University Mainz, Duesbergweg 10-14, 55128 Mainz, Germany

e University of Pardubice, Faculty of Chemical Technology, Studentska 573, 532 10, Pardubice, Czech

Republic

*Corresponding author:

E-mail address : dutkova@saske.sk (E. Dutková)

\begin{abstract}
Chalcopyrite $\mathrm{CuFeS}_{2}$ was shown to be a promising thermoelectric material. Considering thermoelectric efficiency, its relatively high and temperature weakly dependent power factor, economic affordability and ecological benignity is counter balanced by a high lattice thermal conductivity. Thus it is highly desirable to lower the thermal conductivity of thermoelectric chalcopyrite material without deterioration of other thermoelectric characteristics. In our study, we demonstrate that mechanosynthesis followed by appropriate sintering enables to prepare such nanostructured ceramics with a favourable thermoelectric response. Our study shows that mechanosynthesis is a technological route leading to the low-cost synthesis of thermoelectric chalcopyrite ceramics.
\end{abstract}

Keywords: chalcopyrite; nanocomposites; mechanochemistry; thermoelectrics

\section{Introduction}

Chalcopyrite $\mathrm{CuFeS}_{2}$ is a frequently exploited copper-bearing mineral composed of earthabundant, non-toxic, and inexpensive elements. The structure can be regarded as double sphalerite cell with an ordered arrangement of $\mathrm{Cu}$ and $\mathrm{Fe}$ ions in the lattice that are in tetrahedral coordination with sulphur. CuFeS 2 is a narrow-bandgap magnetic semiconductor with a bandgap ( $\left.\mathrm{E}_{\mathrm{g}}\right)$ of $0.53 \mathrm{eV}$ [1]. Iron ions carry magnetic moments, which order antiferromagnetically with Curie temperature of $823 \mathrm{~K}$ [2]. Together with other chalcogenides, chalcopyrite recently attracted the attention of research community as a material interesting for thermoelectrics [3-6]. In thermoelectrics, dimensionality reduction up to several nanometers plays a crucial role $[7,8]$. Size and morphology of grains strongly affect the transport of phonons and charge carriers, which significantly influences thermoelectric performance. In addition to nanostructuring, the focus is also given to point defects and dislocations that can effectively scatter phonons and thus reduce the thermal conductivity. Thus the concept of nanostructuring and defect formation besides doping dominates today in research on thermoelectrics $[9,10]$.

Mechanochemistry using tools of high-energy milling is a scalable and reproducible method for the production of nanograins [11]. Moreover, the mechanochemically synthesized compounds are composed of morphologically inhomogeneous, weakly crystallized and structurally disordered nanoparticles. Based on our previous knowledge, mechanochemical solid-state approach to synthesize chalcogenides proved to be a simple, low-temperature and eco-friendly process [12]. In addition to milling, subsequent hot pressing has to be used in order to obtain the final material with 
the highest density possible, which is very important for nanostructured thermoelectric compounds [13].

In this paper, we applied the novel strategy to synthesize chalcopyrite thermoelectric nanocomposite by combining nanostructuring $\left(\mathrm{CuFeS}_{2}\right.$ prepared from elemental precursors) and nanodisordering (mechanical activation of $\mathrm{CuFeS}_{2}$ mineral) using mechanochemical approach.

\section{Experimental}

The following samples were studied in this work: (i) natural chalcopyrite $\mathrm{CuFeS}_{2}$ (sample labelled "N"), deposit Zhezkazgan, Kazachstan, with the admixture of $0.95 \% \mathrm{SiO}_{2}$ and $2.82 \%$ insoluble residue. This sample was sieved to particle sizes $<200$ micrometers and then treated by mechanical activation, (ii) synthetic chalcopyrite $\mathrm{CuFeS}_{2}$ ("S") synthesized from copper (99\%, Merck, Germany), iron (extra pure, Merck, Germany), and sulphur (99\%, Ites, Slovakia) by mechanochemical synthesis from elements and (iii) chalcopyrite nanocomposite $\mathrm{CuFeS}_{2}$ ("NS") prepared from pure elements as precursors simultaneously co-milled with natural chalcopyrite in a weight ratio 1:1. Mechanical activation (MA) and mechanochemical synthesis (MS) of chalcopyrite were performed in a laboratory planetary ball mill Pulverisette 6 (Fritsch, Germany), details of the synthesis are provided in the Supplementary information (SI).

For phase analysis of chalcopyrite, the X-ray diffraction (XRD) patterns of the powder samples were collected by using a D8 Advance diffractometer (Brucker, Germany) with the CuK $K_{\alpha}$ radiation in the Bragg-Brentano configuration. Transmission electron microscopy (TEM) along with selected area diffraction (SAD) and energy-dispersive X-ray spectroscopy (EDS) were used for characterization of the samples at the nanoscale, details are given in SI. The ${ }^{57} \mathrm{Fe}$ Mössbauer spectroscopy of powder samples was employed to clarify the magnetic ordering in nanoparticles and to identify possible admixtures of other iron-bearing compounds, for experimental details see SI.

The samples for thermoelectric measurements were hot-pressed at $763 \mathrm{~K}$ and $50 \mathrm{MPa}$ for $1 \mathrm{hr}$. The resulting disc-shaped ceramics (diameter $12 \mathrm{~mm}$ and thickness ca $2 \mathrm{~mm}$ ) evinced relative density exceeding $95 \%$ of the theoretical (X-ray) value. During the sintering process, the size of grains increased three times compared to the original powder. The pellets were cut for subsequent analyses by a diamond wire saw. The electrical resistivity, thermoelectric power and thermal conductivity were measured in a temperature range between $3-600 \mathrm{~K}$ via the four-probe method in different setups, which are described in SI.

\section{Results and discussions}

The characterization of the prepared powders is depicted in Fig. 1. The phase analysis based on XRD confirmed that chalcopyrite is the dominating phase with the grain size close to $12 \mathrm{~nm}$ (Fig. 1a). As the ${ }^{57} \mathrm{Fe}$ Mössbauer spectroscopy offers the key insight into the phase composition and magnetic order of iron-based materials, we show our room-temperature Mössbauer spectra in Fig. 1b. The present magnetic order is manifested by the magnetically split components - sextets [14]. Both the MS and MA powder samples exhibit a certain level of magnetic disorder caused by structural defects, which are manifested by asymmetric broadening of the spectral lines (see minor sextets with the decreased hyperfine field in Fig. $1 \mathrm{~b}$ and in Fig. S1 in SI). The low-temperature spectra support the presence of 6-12\% non-magnetic pyrite with similar hyperfine parameters as reported in Ref. [15], and consistent with our XRD (see the reflections marked by diamonds in Fig. 1a). Further, the low-temperature spectra provide evidence that the powder samples contain $\beta$ - or $\gamma$-FeOOH (hyperfine parameters for comparison: $\gamma$-FeOOH [16]), most probably amorphous as the uppermost surface layer of the particles (roughly 3-6\%). The comparison of the room-temperature spectra with those at low temperatures (see Fig. S1 in SI) clearly indicates that magnetic fluctuations occur at ambient temperature in the chalcopyrite phase with the characteristic time scale of the method (i.e. $\left.10^{-10}-10^{-7} \mathrm{~s}\right)$. 
(a)
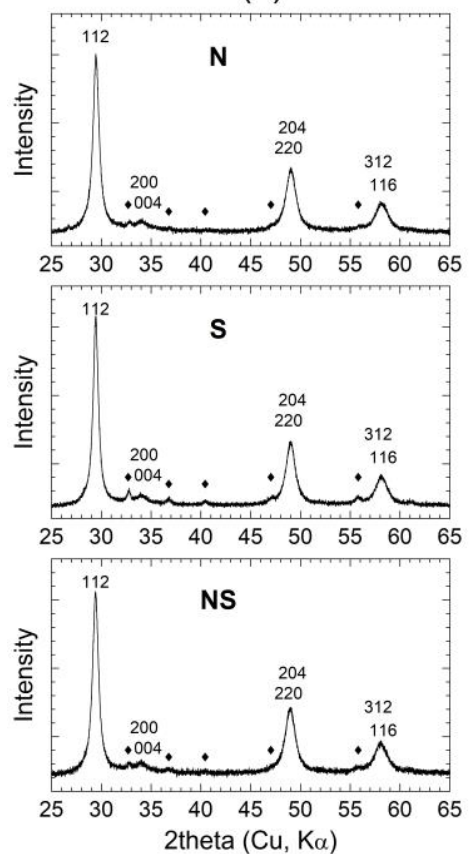

(b)
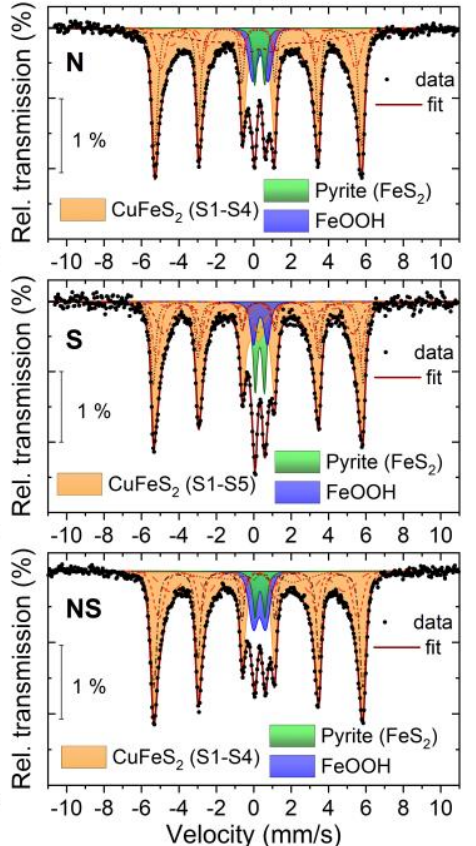

(c)
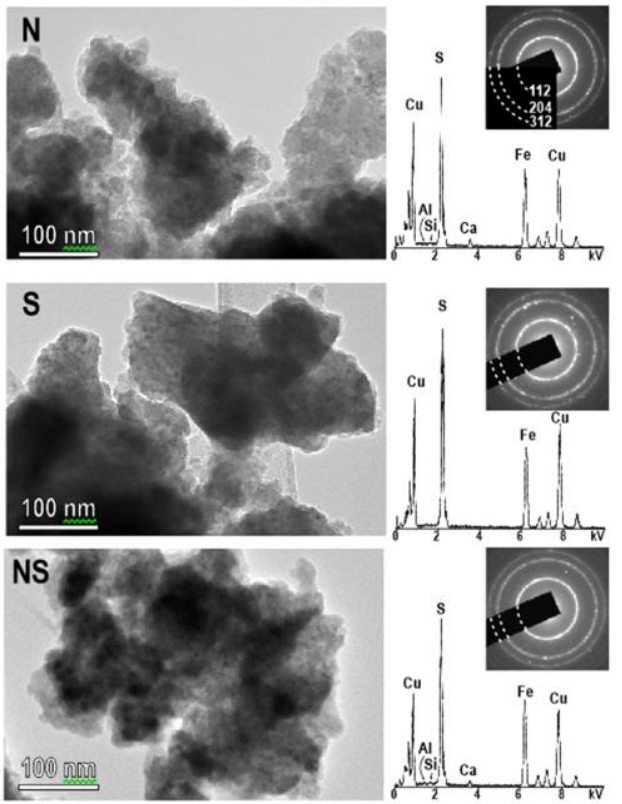

Figure 1: Room-temperature measurements: (a) XRD patterns with reflections of pyrite marked with diamonds, (b) ${ }^{57} \mathrm{Fe}$ Mössbauer spectra and (c) TEM figures with EDS spectra and SAD patterns of powder samples.

The TEM studies (Fig. 1c) confirm that all three samples are composed of agglomerated nanoparticles with crystallite sizes in the $\sim 10-20 \mathrm{~nm}$ range. The three most intense rings in the SAD patterns agree with the three most intense reflections observed in the XRD patterns which correspond to reflections from (112), (204) and (312) lattice planes. The main difference between the samples is in their chemical composition, especially in the $\mathrm{Cu} / \mathrm{Fe}$ ratio (Table 1 ).

Table 1: Chemical composition (molar \%) of the samples determined by TEM/EDS.

\begin{tabular}{l|l|l|l|l} 
Sample & S & Fe & Cu & Cu:Fe \\
\hline N (standard) & $\mathbf{5 0 . 0} \pm \mathbf{1 . 8}$ & $\mathbf{2 5 . 0} \pm \mathbf{0 . 7}$ & $\mathbf{2 5 . 0} \pm 1.7$ & $\mathbf{1 . 0} \pm \mathbf{0 . 1}$ \\
S & $49.7 \pm 1.7$ & $22.9 \pm 0.3$ & $27.0 \pm 1.5$ & $1.2 \pm 0.1$ \\
NS & $48.4 \pm 2.5$ & $26.7 \pm 1.0$ & $24.9 \pm 1.9$ & $0.9 \pm 0.1$
\end{tabular}

In addition to three main elements $(\mathrm{Cu}, \mathrm{Fe}$ and $\mathrm{S})$, the samples prepared from natural chalcopyrite $(\mathrm{N}, \mathrm{NS})$ contain a small amounts of impurity elements like $\mathrm{Mg}, \mathrm{Al}, \mathrm{Si}$ and $\mathrm{Ca}$ (see EDS spectrum in Fig. 1c). For the sample (S) other impurities were not detected; however, the sample contains a lower amount of $\mathrm{Fe}$ and higher amount of $\mathrm{Cu}$ in comparison to samples $\mathrm{N}$ and NS. The excess copper indicates that the core of the chalcopyrite particles is formed by $\mathrm{Cu}$-rich amorphous sulfide. This can be understood by supposing that $\mathrm{Cu}$ and $\mathrm{S}$ initially react to CuS [17], whereas Fe is more slowly incorporated into the structure to form the targeted chalcopyrite phase. The $\mathrm{Cu}: \mathrm{Fe}$ ratio in this sample thus exceeds the ideal ratio. Interestingly, the sample prepared by co-milling the natural mineral with the elements (sample NS) contains slight excess of Fe with respect to $\mathrm{Cu}$, which indicates that the presence of natural chalcopyrite stimulates the formation of additional chalcopyrite from the added elements. Most importantly, similar thermoelectric properties of both $\mathrm{N}$ and NS samples (see Fig. 2) point to the same stoichiometry/doping of both chalcopyrite phases and, in this respect, the excess of iron in analyzed nanoparticles can be explained by presuming excess of 
amorphous binary Fe-S or Fe-O phases occurring likely on the surface of chalcopyrite nanoparticles (see the analysis of Mössbauer spectra at low temperatures in $\mathrm{SI}$ ).
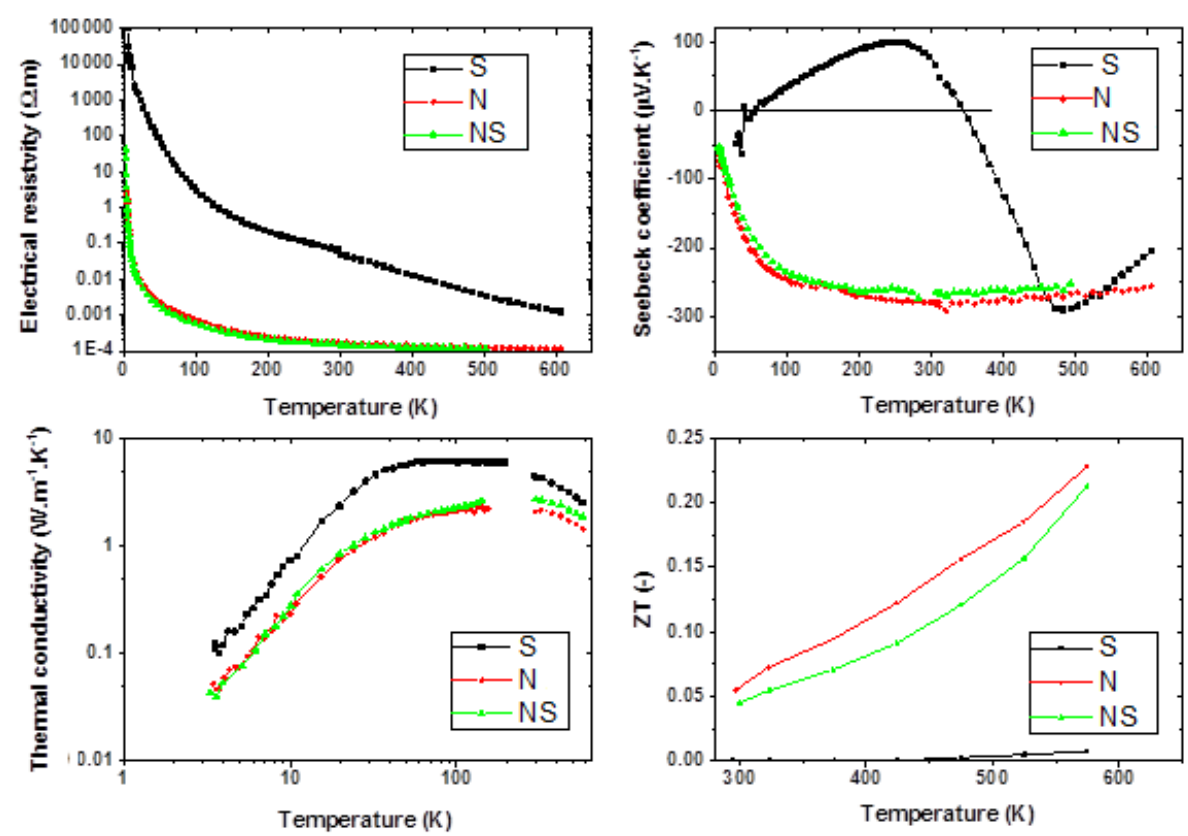

Figure 2: Temperature dependence of the basic thermoelectric characteristics (specific electrical resistance, Seebeck coefficient, thermal conductivity and figure of merit (ZT) of studied samples.

Table 2: Figure of merit $Z T$ of the samples at different temperatures.

\begin{tabular}{c|c|c|c} 
Sample & $Z T$ at $200 \mathrm{~K}$ & $Z T$ at $300 \mathrm{~K}$ & $Z T$ at $500 \mathrm{~K}$ \\
\hline $\mathrm{S}$ & 0 & 0 & 0.004 \\
$\mathrm{~N}$ & 0.03 & 0.06 & 0.17 \\
$\mathrm{NS}$ & 0.03 & 0.04 & 0.14
\end{tabular}

We summarize the essential thermoelectric characteristics of studied nano-chalcopyrites in Fig. 2 and Table 2. The sample $S$ exhibits high electrical resistivity and Seebeck coefficient changing the sign. -These characteristics indicate intrinsic state, which might correspond to ideal stoichiometry almost free of vacancies or substitutional defects. The low concentration of point defects (acceptors and donors), in accordance with varying Seebeck coefficient, corresponds to an acceptor level accounting for p-type conductivity at low temperatures while a donor defect accounts for n-type conductivity at higher temperatures. The other two samples ( $N$ and NS) exhibit robust $n$-type character complemented by low electrical resistivity, which reflects the natural tendency to minute Fe:Cu over stoichiometry leading to electron-like doping with highly mobile electrons, analogous to Ref. [18]. Further modification of electronic properties can be done via co-milling of natural chalcopyrite with the addition of $\mathrm{Cu}$, Fe or $\mathrm{S}$ (or binaries) in appropriate amounts but such work exceeds the scope of present article. The important positive impact of the nanostructuring is highlighted by a comparison of the thermoelectric characteristics with that of ceramics based on resintered mineral which both (i) exhibit similar Seebeck coefficients (see SI) in connection with (ii) the significantly lowered thermal conductivity (Fig. 2c) that enhances the figure of merit for nanostructured ceramics (Fig. 2d).

As the essential advantage of nanostructured samples lies in reduced thermal conductivity, we attempted to model the thermal conductivity supposing the "classical" Callaway model [19]. In this respect, the thermal conductivity of the non-milled natural chalcopyrite with the grain size in micrometers and sintered by the identical procedure as the other two studied nanopowders was 
successfully modeled by assuming the three basic scattering mechanisms: grain boundary, point defect and Umklapp scattering with characteristic parameters similar as in ref. [18] (see SI). In the case of the samples containing synthetic chalcopyrite (S, NS), this approach was not sufficient and another $\mathrm{T}^{2}$-dependent low-temperature scattering mechanism had to be added (for details see $\mathrm{SI}$ ). This mechanism likely corresponds to scattering on dislocation strain fields, which are caused by the highly energetic milling process [20].

\section{Acknowledgements}

Financial support from the Czech Science Foundation (project 18-12761S), the Slovak Research and Development Agency (project APVV-18-0357) and the Slovak Grant Agency (projects VEGA $2 / 0044 / 18,2 / 0065 / 18$ ) was greatly appreciated. The work was further supported by Operational Programme Research, Development and Education financed by European Structural and Investment Funds and the Czech Ministry of Education, Youth and Sports (project SOLID21 CZ.02.1.01/0.0/0.0/16_019/0000760). A.M. and V.K. acknowledge funding by the Carl-Zeiss Foundation.

\section{Appendix A Supplementary data}

\section{References}

[1] Habashi F. Chalcopyrite its chemistry and metallurgy. McGraw-Hill, New York 1978.

[2] Teranishi T. Magnetic and electric properties of chalcopyrite. J Phys Soc Jpn. 1961;16:1881-7.

[3] Suekuni K, Takabatake T. Research Update: Cu-S based synthetic minerals as efficient thermoelectric materials at medium temperatures. Apl Materials. 2016;4.

[4] Shi YX, Sturm C, Kleinke H. Chalcogenides as thermoelectric materials. J Solid State Chem. 2019;270:273-9.

[5] Hebert S, Berthebaud D, Daou R, Breard Y, Pelloquin D, Guilmeau E, et al. Searching for new thermoelectric materials: some examples among oxides, sulfides and selenides. Journal of Physics-Condensed Matter. 2016;28:013001.

[6] Powell AV. Recent developments in earth-abundant copper-sulfide thermoelectric materials. J Appl Phys. 2019;126:100901.

[7] Dresselhaus MS, Chen G, Tang MY, Yang RG, Lee H, Wang DZ, et al. New directions for low-dimensional thermoelectric materials. Adv Mater. 2007;19:1043-53.

[8] Heremans JP, Dresselhaus MS, Bell LE, Morelli DT. When thermoelectrics reached the nanoscale. Nature Nanotechnology. 2013;8:471-3.

[9] Biswas K, He JQ, Blum ID, Wu CI, Hogan TP, Seidman DN, et al. High-performance bulk thermoelectrics with all-scale hierarchical architectures. Nature. 2012;489:414-8.

[10] He JQ, Kanatzidis MG, Dravid VP. High performance bulk thermoelectrics via a panoscopic approach. Mater Today. 2013;16:166-76.

[11] Baláž, P, Mechanochemistry in Nanoscience and Minerals Engineering, Springer: Berlin Heidelberg; 2008.

[12] Baláž P, Baláž M, Achimovičová M, Bujňáková Z, Dutková E. Chalcogenide mechanochemistry in materials science: insight into synthesis and applications (a review). Journal of Materials Science. 2017;52:11851-90.

[13] Lan YC, Minnich AJ, Chen G, Ren ZF. Enhancement of thermoelectric figure-of-merit by a bulk nanostructuring approach. Adv Funct Mater. 2010;20:357-76.

[14] Piekoszewski J, Suwalski, J., Ligenza, S. Mössbauer effect study in chalcopyrite. Phys Status Solidi. 1968;29:K99-K101.

[15] Montano PA, Seehra MS. Magnetism of iron pyrite $\left(\mathrm{FeS}_{2}\right)$ - a Mössbauer study in an external magnetic-field. Solid State Commun. 1976;20:897-8. 
[16] Johnson, CE. Antiferromagnetism of $\gamma$ FeOOH: A Mössbauer effect study, J. Phys. C Solid State Phys. 1969; 2:1996-2002.

[17] Dutková, E, Bujňáková, Z, Kováč, J, Škorvánek, I, Sayagues, MJ, Zorkovská, A, Kováč, J, Baláž, P, Adv. Powder Technol. 2018;29:1820-1826.

[18] Li YL, Zhang TS, Qin YT, Day T, Snyder GJ, Shi X, et al. Thermoelectric transport properties of diamond-like $\mathrm{Cu}_{1-\mathrm{x}} \mathrm{Fe}_{1+\mathrm{x}} \mathrm{S}_{2}$ tetrahedral compounds. J Appl Phys. 2014;116.

[19] Callaway J. Model for lattice thermal conductivity at low temperatures. Physical Review. 1959;113:1046-51.

[20] Hanus R, Garg A, Snyder GJ. Phonon diffraction and dimensionality cross over in phonon-interface scattering. Communications Physics. 2018;1:78. 